\title{
Vibrations in MagAO: Resonance sources identification and first approaches for modeling and control
}

\author{
Javier Garcés ${ }^{\mathrm{a}}$, Sebastián Zúñiga ${ }^{\mathrm{a}}$, Laird Close $^{\mathrm{b}}$, Jared Males ${ }^{\mathrm{b}}$, Katie Morzinski ${ }^{\mathrm{b}}$, Pedro \\ Escárate $^{\mathrm{a}}$, Mario Castro ${ }^{\mathrm{a}}$, José Marchioni ${ }^{\mathrm{a}}$, and Diego Rojas ${ }^{\mathrm{a}}$ \\ aAC3E, Universidad Técnica Federico Santa María, Avenida España 1680, Valparaíso, Chile

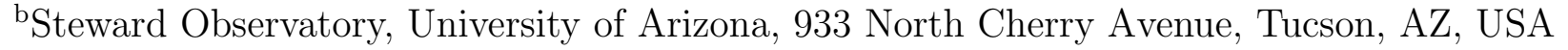

\begin{abstract}
The Magellan Telescope Adaptive Optics System (MagAO) is subject to resonance effects induced by elements within the system instrumentation, such as fans and cooling pumps. Normalized PSDs are obtained through frequency-based analysis of closed-loop on-sky data, detecting and measuring vibration effects. Subsequently, a space-state model for the AO loop is obtained, using a standard AO loop scheme with an integrator-based controller and including the vibration effects as disturbances. Finally, a new control alternative is proposed, focusing on residual phase variance minimization through the design and simulation of an optimal LQG control approach.
\end{abstract}

Keywords: Adaptive optics, MagAO, vibrations, modeling, control.

\section{INTRODUCTION}

The 6.5m Magellan Telescope Adaptive Optics System (MagAO), ${ }^{1}$ designed by the MagAO Team from the University of Arizona, is noticeable for being the first AO system in the southern hemisphere based on an adaptive secondary mirror, and the first AO system able to provide high quality diffraction-limited images (0.6 to 1.0 microns), reaching resolutions up to $20[\mathrm{mas}] .^{2}$

The system, however, is not exempt from additional factors which are able to reduce the acquired data's reliability. These factors include resonance effect from the telescope itself, as well as the effect of certain instrumentation elements. It is of great interest to identify these perturbation sources, to be able to recognize precisely how the instrumentation elements are influencing the data captured by the sensors.

This research work has been conducted using closed-loop on-sky data taken during observing runs of the MagAO Team at the Clay Telescope in Las Campanas Observatory, in order to study (under different operation conditions) the frequency-domain data captured by sensors of the MagAO system. ${ }^{3,4}$ Data is obtained from both the VisAO science camera ${ }^{5}$ and the waveform sensor's modal amplitudes.

Spectral analysis of sampled data allows an extension of the research work into obtaining mathematical models to describe the system. For this process, a standard AO loop scheme is used, ${ }^{6}$ taking into account the presence of disturbances due to turbulence and vibration components, to obtain a space-state model for the AO loop. Autoregressive models ${ }^{7}$ are used to describe the temporal correlation of the turbulence and vibration phases. A modal control approach is employed, to be able to evaluate the effects of vibrations on each mode separately (vibrations primarily affect tip/tilt modes).

The next step is to improve the system's performance by designing a control scheme for the proposed model. Previous work has been made studying the design of LQG control or frequency-based control for specific AO systems. ${ }^{6}$ With a state-space model, LQG control can be implemented on simulation and compared to the integrator-based controller alternative, and a predictive control approach (MPC) is analysed for the system.

Further author information:

Javier Garcés: E-mail: javier.garces@alumnos.usm.cl.

Adaptive Optics Systems V, edited by Enrico Marchetti, Laird M. Close, Jean-Pierre Véran, Proc. of SPIE Vol. 9909, 99093G (C) 2016 SPIE $\cdot$ CCC code: 0277-786X/16/\$18 · doi: 10.1117/12.2232777 


\section{VIBRATIONS IN MAGAO}

\subsection{On-sky Data}

This research work was conducted using closed-loop on-sky data taken during the 2014b observing run by the University of Arizona MagAO Team, the night of October 31st, 2014, at the Clay Telescope in Las Campanas Observatory.

During this night, real-time data has been captured from two sensors: the VisAO Science Camera and the Pyramidal Wavefront Sensor.

1. $\mathrm{X}$ and $\mathrm{Y}$-axis movement of the brightest pixel captured by the CCD camera is registered, at a capture rate of $\sim 42[\mathrm{~Hz}]$. This set of data is hereby referred to as "CCD data".

2. Wavefront's modal amplitudes from the first 200 modes are registered by the PWFS. The sensor's capture rate is $\sim 990[\mathrm{~Hz}]$, however only one in three measurements are stored, thus giving us a capture rate of $\sim$ $330[\mathrm{~Hz}]$. Only tip and tilt modes will be used in this analysis, since they are most subject to vibration components. This set of data is hereby referred to as "WFS data".

Tthe system is tested under different conditions, and for each test, CCD data and WFS data is recorded simultaneously, within a time interval of approximately one minute. Specifically, the status of five of the system instrumentation elements are tested: primary fans, primary pump, louvers, VisAO fans and bayside stages.

Table 1 presents the system element's status during six of the tests conducted during the observing run.

Table 1: System element status during tests carried out on the 2014b run.

\begin{tabular}{|cccccc|}
\hline & Primary Fans & Primary Pump & Louvers & VisAO Fans & Bayside Stages \\
\hline Test A & Off & Off & Closed & On & On \\
\hline Test B & On & Off & Closed & On & On \\
\hline Test C & On & On & Closed & On & On \\
\hline Test D & On & On & Open & On & On \\
\hline Test E & Off & Off & Closed & Off & On \\
\hline Test F & Off & Off & Closed & On & Off \\
\hline
\end{tabular}

Additionally, one set of CCD and WFS data from the previous observing run (2014a) is available, with the same system element's status from Test A.

Power spectral densities (PSDs) of the time-domain data captured by the sensors are obtained, using the Welch method. ${ }^{8}$ PSDs from the CCD and WFS data are obtained, hereby referred to as "CCD PSDs" and "WFS PSDs". Due to each of the datasets' capture rate, frequency components up to $21[\mathrm{~Hz}]$ can be analysed with the CCD PSDs, while the WFS PSDs are limited up to $165[\mathrm{~Hz}]$.

\subsection{PSD Normalization Process}

It is of interest to obtain RMS values (root-integrated-power) in units of [mas], for each vibration component as well as for the total PSD.

Since we can relate CCD camera pixel length to its corresponding angular resolution value in [mas], it is possible to obtain CCD PSDs measurable in units of $\left[\mathrm{mas}^{2} / \mathrm{Hz}\right]$. Then, a normalization process is necessary to obtain WFS PSDs in units of $\left[\mathrm{mas}^{2} / \mathrm{Hz}\right]$, based on the CCD PSDs.

In previous work, ${ }^{3,4}$ WFS PSDs has been normalized by amplifying them in order to obtain the same RMS value as the CCD PSDs within a range of 15 to $21[\mathrm{~Hz}]$, assuming lower frequencies to be subject to aliasing.

Results here presented take a different approach at normalizing the WFS data. Instead, time-domain WFS data is amplified to match the variance of the time-domain CCD data, therefore, matching the average power of both signals. WFS PSDs obtained from the amplified WFS datasets are measured in units of $\left[\mathrm{mas}^{2} / \mathrm{Hz}\right]$. The numerical results obtained differ from the ones previously presented. 


\subsection{Inherent Vibration Components}

PSDs from WFS data of 2014a and 2014b (Test A) observing runs are presented in Figures 1 and 2 respectively.
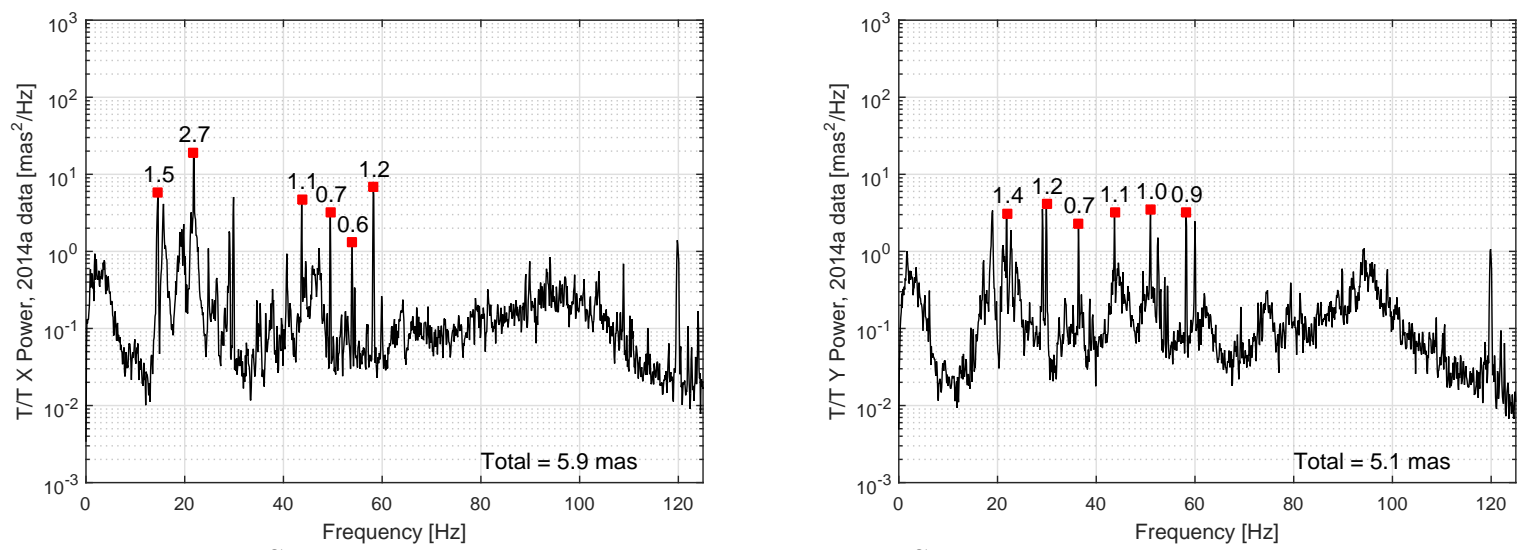

Figure 1: Vibration PSDs measured in closed-loop on-sky. WFS data observed during 2014a observing run. WFS data is normalized matching CCD data variance. Peaks are labeled with RMS values in [mas]. Total RMS value in [mas] is presented. Left: $\mathrm{X}$ axis. Right: $\mathrm{Y}$ axis.
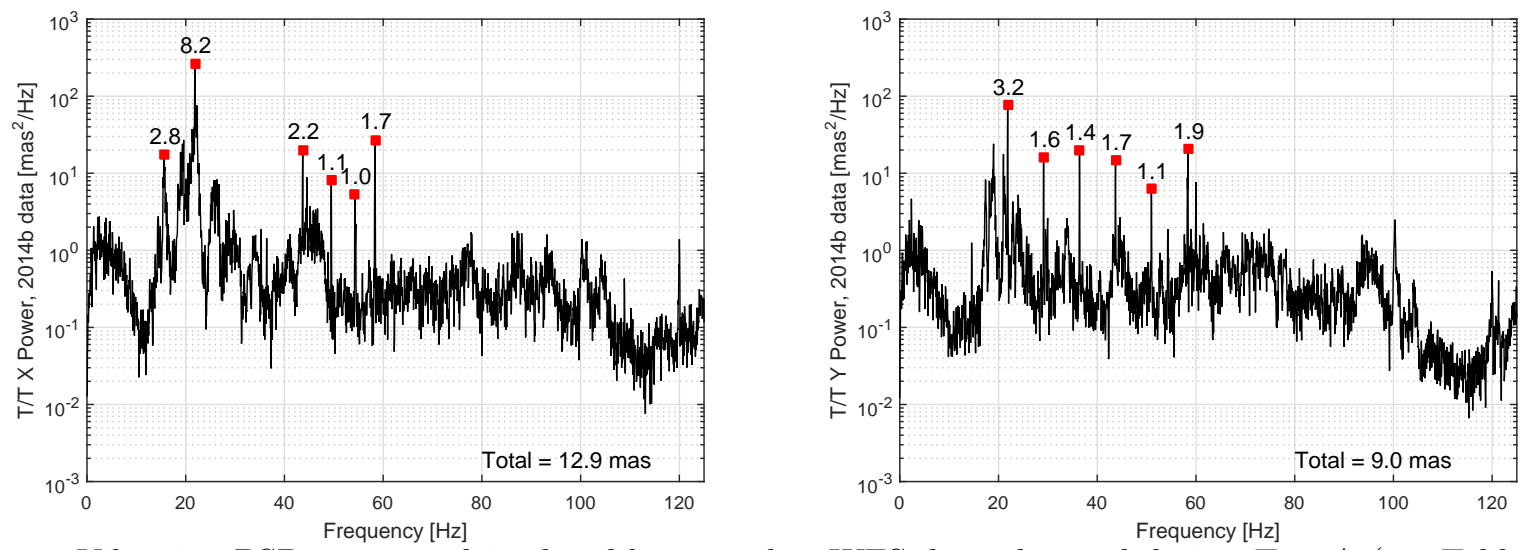

Figure 2: Vibration PSDs measured in closed-loop on-sky. WFS data observed during Test A (see Table 1) of 2014b observing run. WFS data is normalized matching CCD data variance. Peaks are labeled with RMS values in [mas]. Total RMS value in [mas] is presented. Left: $\mathrm{X}$ axis. Right: $\mathrm{Y}$ axis.

Both sets of data presented here are "all-off" data, that is, the main instruments (primary fans, primary pump, louvers) are off or closed, so no resonance components from them are present.

RMS values for the 2014a WFS PSDs are 5.8 [mas] and 5.1 [mas] for X and Y axis respectively. Since the night of the 2014b observing run was subject to worse weather conditions and astronomical seeing, higher RMS values are expected. The RMS values obtained from the 2014b WFS PSDs (Figure 2) are 12.9 [mas] and 9.0 [mas] for $\mathrm{X}$ and $\mathrm{Y}$ axis.

However, independently of these conditions, the vibration peaks on both sets of PSDs coincide. This is evidence that there are inherent vibrations on the system. These vibration peaks are present in all WFS data. They can be attributed to different factors, such as the telescope's resonance frequency's harmonics (it is known the telescope presents resonance at $\sim 7[\mathrm{~Hz}],{ }^{2}$ and equidistant vibration peaks in the Y-axis data, multiples of the frequency value $7.28[\mathrm{~Hz}]$, are observed) or the observatory's electrical grid (peaks near $60[\mathrm{~Hz}]$ and $120[\mathrm{~Hz}]$ ). 


\subsection{Resonance Sources Identification}

Instrumentation influences on the observed sensor data can be analysed by observing differences between WFS PSDs. For each instrument, we compare the WFS PSDs obtained from two tests which differ only on the specific instrument's state (see Table 1). The frequency peaks present in only the dataset where the instrument is working are contributed to the specific instrument.

Primary Fans Effect By comparing WFS PSDs from Tests A and B, we can identify the influence of the primary mirror cell fans. The ratio between WFS PSDs from Test B (primary fans on) and Test A (primary fans off) is obtained. WFS PSD from Test A is smoothed in order to counter noise effects. Peaks observed in the PSD ratio correspond to frequency components caused by this instrument.
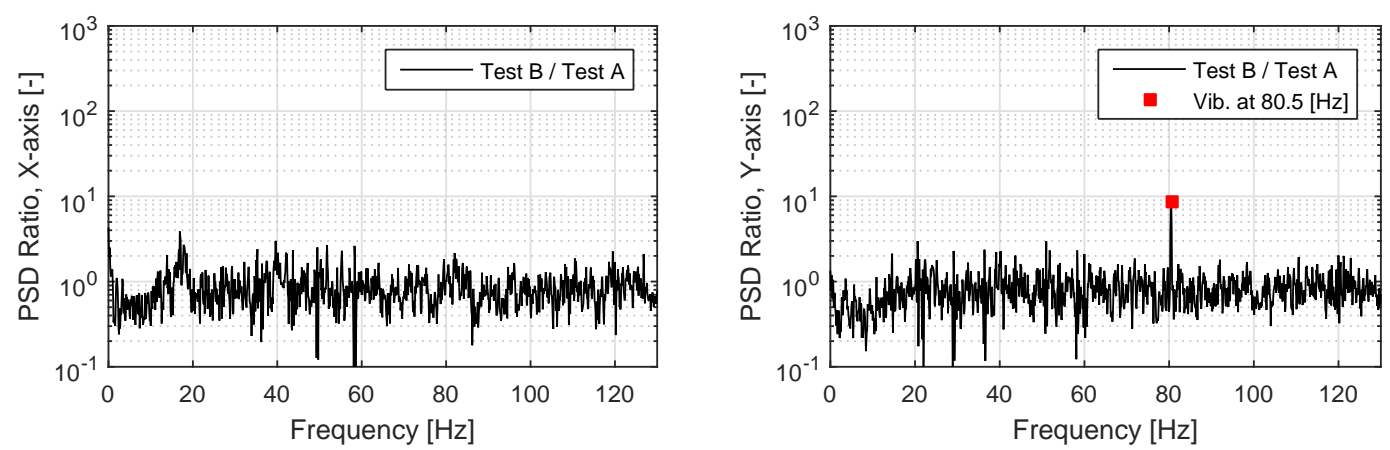

Figure 3: Identification of vibrations induced by primary fans, on the sensor's captured data.

There is a vibration peak in the Y-axis WFS PSD, at $80.5[\mathrm{~Hz}]$. To measure the RMS value contributed by this instrument at the resonance frequency, root-integrated-power is calculated for both tests. Figure 4 presents the Y-axis PSDs for Tests A and B near $80.5[\mathrm{~Hz}]$.

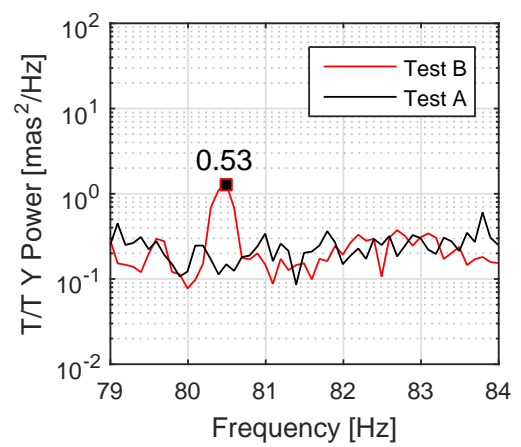

Figure 4: Vibrations induced by primary fans in Y-axis WFS data PSD. RMS value of resonance contribution is included for each peak.

By integrating between $80[\mathrm{~Hz}]$ and $81[\mathrm{~Hz}]$, a RMS value (root-integrated-power) of 0.43 [mas] is obtained for Test A and 0.68 [mas] for Test B. Therefore, this vibration contributes with a RMS value of 0.53 [mas]. This value is presented in Figure 4. 
Primary Pump Effect WFS PSDs from Tests B and C are compared, following the same method presented for the primary fans, in order to analyse the effect of the primary mirror cell glycol cooling pump.
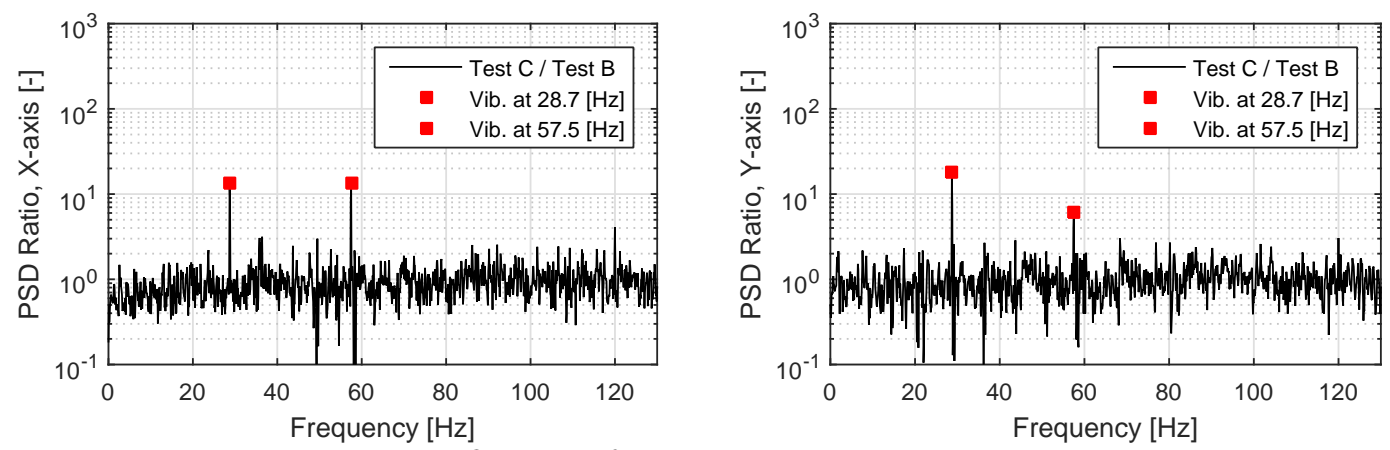

Figure 5: Identification of resonances induced by primary pump.

In this case, there are vibration peaks present in both the $\mathrm{X}$-axis and the $\mathrm{Y}$-axis WFS PSDs.

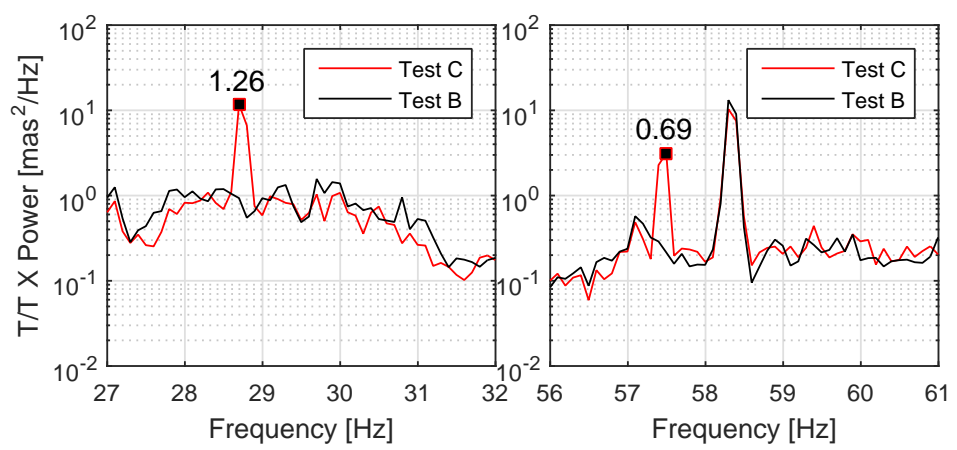

Figure 6: Vibrations induced by primary pump in X-axis WFS data PSD. RMS value of resonance contribution is included for each peak.

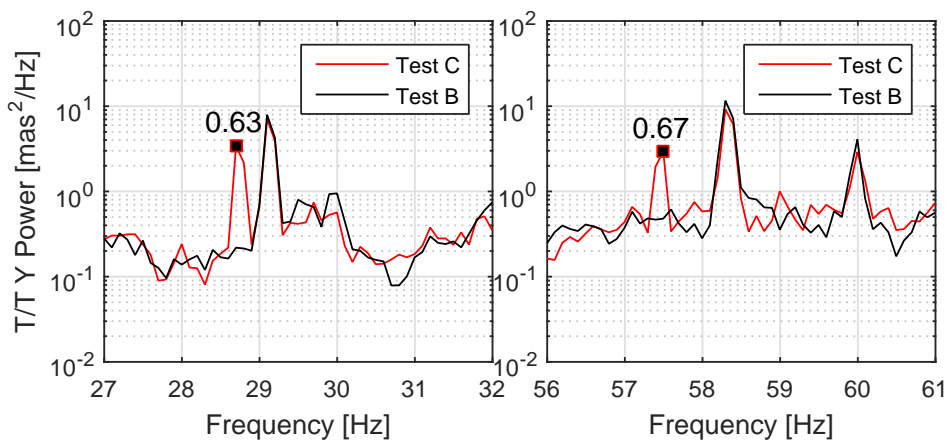

Figure 7: Vibrations induced by primary pump in Y-axis WFS data PSD. RMS value of resonance contribution is included for each peak.

It is possible to attribute the second vibration peak (at $57.5[\mathrm{~Hz}]$ ) as the second harmonic for the first vibration peak (at $28.7[\mathrm{~Hz}])$. 
Louvers Effect WFS PSDs from Tests C and D are compared, following the same method presented before.
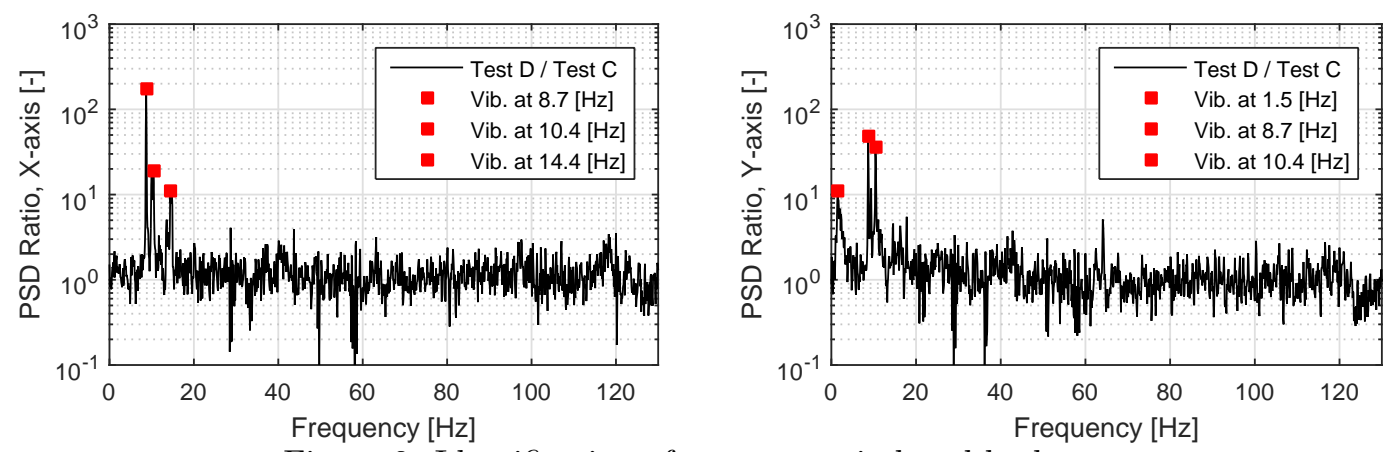

Figure 8: Identification of resonances induced by louvers.

There are vibration peaks in the $\mathrm{X}$-axis and the $\mathrm{Y}$-axis WFS data.

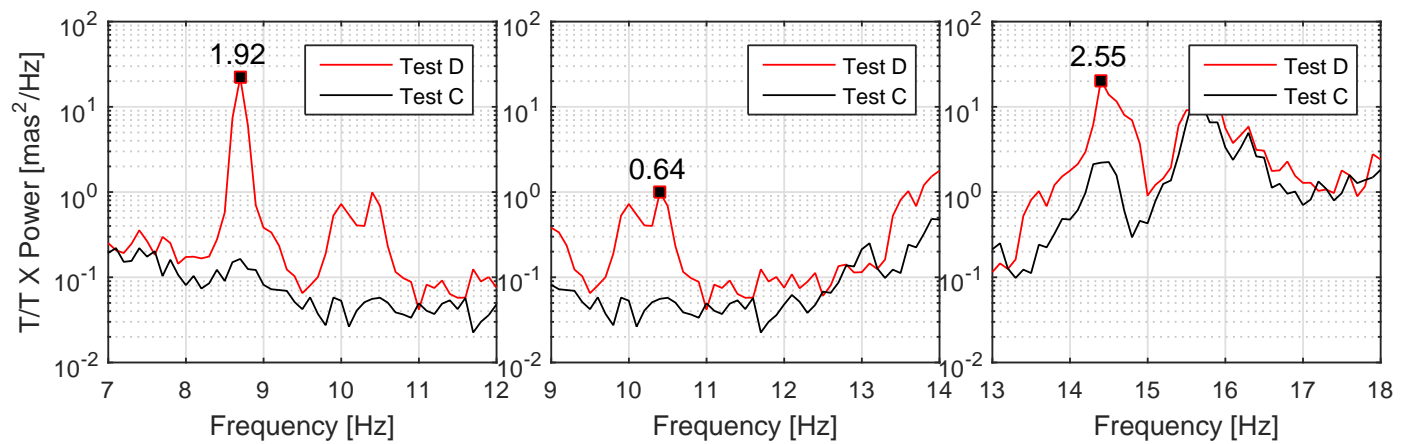

Figure 9: Vibrations induced by louvers in X-axis WFS data PSD. RMS value of resonance contribution is included for each peak.

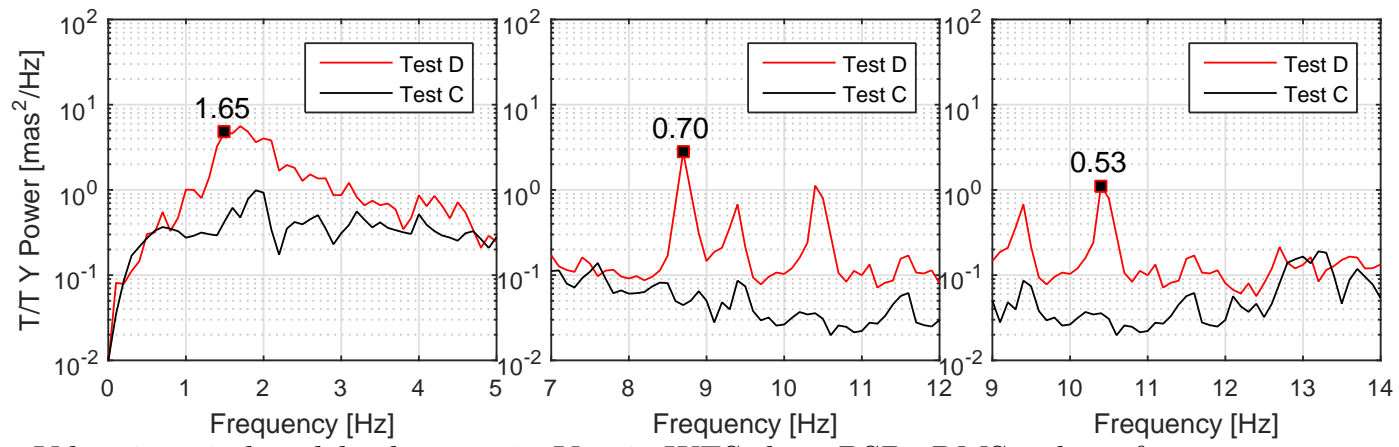

Figure 10: Vibrations induced by louvers in Y-axis WFS data PSD. RMS value of resonance contribution is included for each peak.

The louvers have the strongest influence in WFS PSDs among the instrumentation of interest.

VisAO Fans and Bayside Stages Effect Tests A and E are compared to analyse the VisAO Fans influence, while Tests A and F are compared to analyse the Bayside Stages influence. However, no vibration peaks were detected. Therefore, these instruments do not induce vibrations. 


\section{MODELING FOR MAGAO}

\subsection{Model Structure}

The standard structure for an AO closed loop ${ }^{6}$ consisting of a wavefront sensor (WFS), a deformable mirror $(\mathrm{DM})$ and a controller, is presented in Figure 11.

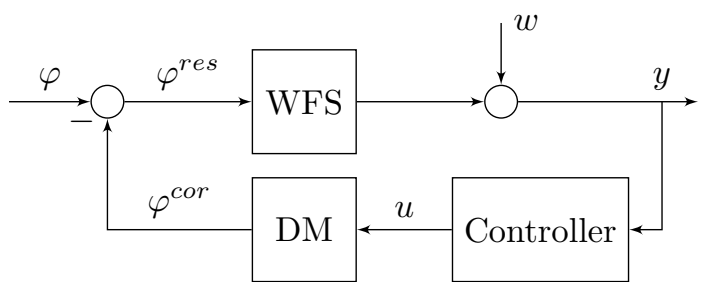

Figure 11: Block diagram for AO closed-loop system.

A modal control approach will be used. For each of the wavefront modes, a different control design can be implemented, following the structure presented.

In Figure 11, $\varphi, \varphi^{\text {cor }}$ and $\varphi^{\text {res }}$ represent the modal amplitudes for total disturbance phase, correction phase and residual phase, respectively. Total disturbance $\varphi$ is defined as the sum of the contributions from the turbulence signal $\varphi^{\text {tur }}$ and several vibration signals $\varphi^{v i b}$.

$$
\varphi_{k}=\varphi_{k}^{t u r}+\varphi_{k}^{v i b, 1}+\cdots+\varphi_{k}^{v i b, n}
$$

The WFS measurement process and the DM correction process are assumed to be linear, and are modeled by a scaling factor and a time delay. Measurement white noise $w$ is also included.

$$
\begin{aligned}
y_{k} & =D \varphi_{k-1}^{r e s}+w_{k} \\
\varphi_{k}^{c o r} & =N u_{k-1}
\end{aligned}
$$

Then, the resulting model is presented in Figure 12.

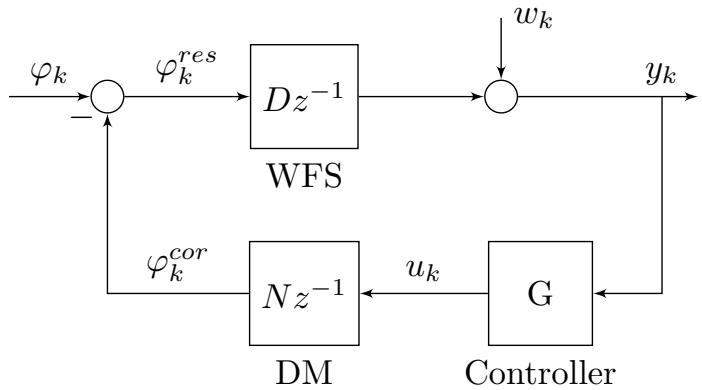

Figure 12: Block diagram for AO closed-loop system, with linear models for WFS and DM. 


\subsection{Controller Modeling}

The classical integrator is the most common controller in AO applications. ${ }^{6}$ As such, the MagAO loop is modeled with an integrator-based controller.

$$
G(z)=\frac{g}{1-a z^{-1}}
$$

While $a$ is generally unity, the controller gain $g$ is adjusted according to noise and performance requirements. This value can be chosen by taking a minimum variance control approach, ${ }^{9}$ designing $g$ to minimize variance of residual slopes $\varphi^{\text {res }}$.

The residual phase modal amplitude $\varphi^{\text {res }}$ is determined by the two inputs of the AO loop: total disturbance phase modal amplitude $\varphi$ and measurement noise $w$.

$$
\varphi^{r e s}(j \omega)=E(j \omega) \varphi(j \omega)+H(j \omega) w(j \omega)
$$

The rejection transfer function $E(z)$ and the noise transfer function $H(z)$ are defined by

$$
E(z)=\frac{1-a z^{-1}}{1-a z^{-1}+D N g z^{-2}} \quad H(z)=\frac{-N g z^{-1}}{1-a z^{-1}+D N g z^{-2}}
$$

From equation (5), it is possible to express the PSD of $\varphi^{\text {res }}$ as a function of PSDs of $\varphi$ and $w$. PSDs are denoted by $\langle|\cdot|\rangle$.

$$
\left\langle\left|\varphi^{r e s}(j \omega)\right|^{2}\right\rangle=|E(j \omega)|^{2}\left\langle|\varphi(j \omega)|^{2}\right\rangle+|H(j \omega)|^{2}\left\langle|w(j \omega)|^{2}\right\rangle
$$

By Parseval's theorem, the residual phase variance $\sigma_{\text {res }}^{2}$ is given by the corresponding PSD's integration.

$$
\sigma_{\text {res }}^{2}=\int\left\langle\left|\varphi^{r e s}(j \omega)\right|^{2}\right\rangle d \omega
$$

Then, one can design the controller gain $g$ to obtain the transfer functions $E(j \omega)$ and $H(j \omega)$ which minimizes: ${ }^{10}$

$$
\sigma_{\text {res }}^{2}=\int|E(j \omega)|^{2}\left\langle|\varphi(j \omega)|^{2}\right\rangle d \omega+\int|H(j \omega)|^{2}\left\langle|w(j \omega)|^{2}\right\rangle d \omega
$$

In order to obtain the optimal value for $g$, estimates for the total disturbance phase's and measurement noise's PSDs and a model for the AO loop (that is, values for $D$ and $N$ ) are necessary.

In the following modeling process, we will define $a=1$ for the controller and $D=N=1$ for the AO loop. Then, the closed loop is stable for values between 0 and 1 for the controller gain $g$. We will set the value of $g$ to 0.5 .

\subsection{Disturbance Phase Estimation}

As previously stated, we will define the static gains from the WFS and DM processes as unity $(N=D=1)$, as well as $a=1$ and $g=0.5$ for the controller. Aditionally, we will use only the WFS PSDs from Test A, since all analysis here presented is analogous for other measurement tests. Finally, only the X-axis PSD will be modeled.

MagAO WFS's on-sky data captured during the 2014b run correspond to the output $y$ of the AO loop. Then, it is possible to estimate the PSD of total disturbance phase $\left\langle|\varphi(j \omega)|^{2}>\right.$ from the output's PSD $\left\langle|y(j \omega)|^{2}\right\rangle$.

$$
\left\langle|y(j \omega)|^{2}\right\rangle=\left|H_{y \varphi}(j \omega)\right|^{2}\left\langle|\varphi(j \omega)|^{2}\right\rangle+\left|H_{y w}(j \omega)\right|^{2}\left\langle|w(j \omega)|^{2}\right\rangle
$$

where the transfer functions are defined by

$$
H_{y \varphi}(z)=\frac{D z^{-1}\left(1-a z^{-1}\right)}{1-a z^{-1}+D N g z^{-2}} \quad H_{y w}(z)=\frac{1-a z^{-1}}{1-a z^{-1}+D N g z^{-2}}
$$


Measurement noise PSD. From equation (10), it is possible to consider the output PSD as being defined by a disturbance phase PSD component $\left(\left|H_{y \varphi}(j \omega)\right|^{2}\left\langle|\varphi(j \omega)|^{2}\right\rangle\right)$ and a measurement noise PSD component $\left(\left|H_{y w}(j \omega)\right|^{2}\left\langle|w(j \omega)|^{2}\right\rangle\right)$.

The measurement noise PSD component is defined by the noise variance $\left(\sigma_{w}^{2}\right)$. An upper bound for the noise variance is to define it such that the power at high frequencies on the output PSD is determined by its noise PSD component. An estimate for the measurement noise PSD component, choosing the upped bound for the noise variance, is presented in Figure 13a.

Disturbance Phase PSD. From equation (10) we can obtain an estimate of the total disturbance phase PSD. The measurement noise PSD component is considered to be comparatibly small, so the disturbance phase PSD is estimated by

$$
\left\langle|\varphi(j \omega)|^{2}\right\rangle=\frac{\left\langle|y(j \omega)|^{2}\right\rangle}{\left|H_{y \varphi}(j \omega)\right|^{2}}
$$

The disturbance phase PSD estimate is presented in Figure 13b.

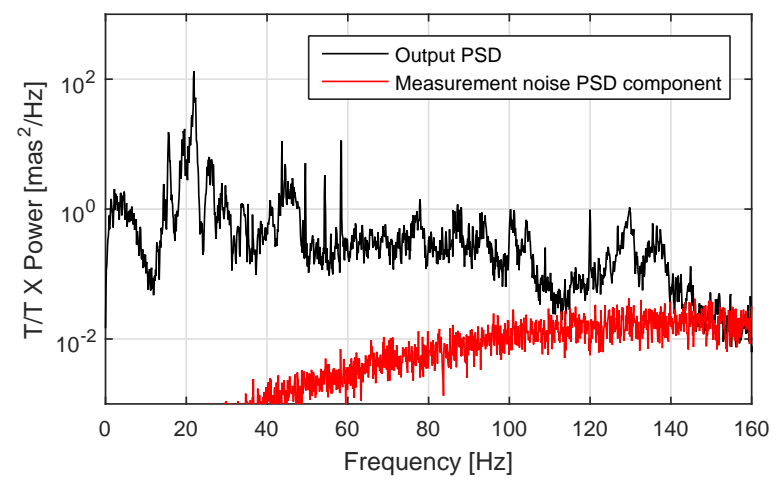

(a) Estimate of measurement noise PSD component.

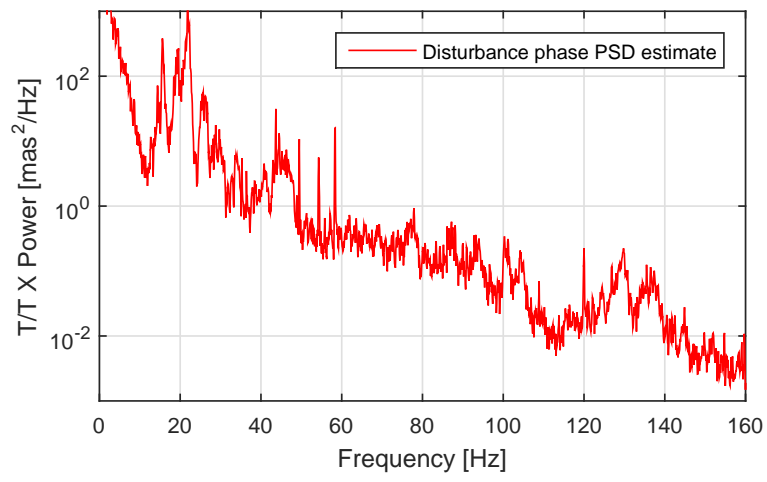

(b) Estimate of total disturbance phase PSD.

Figure 13: Output PSD components from measurement noise and total disturbance phase.

Even though we have obtained estimates of the disturbance phase PSD and the measurement noise PSD, these are not sufficient to compute the optimal value for controller gain according to (9). The optimal design of controller gain $g$ needs precise estimates for the disturbance phase PSD and the measurement noise PSD at a greater frequency range, as well as reliable values for the WFS and DM static gains $(D$ and $N)$.

\subsection{Disturbance Phase Modeling}

A modeling alternative widely used to describe temporal correlation of turbulence and vibration phases are autoregresive models (AR). ${ }^{7}$ Studies have shown that a second order model (AR2) for each phase leads to good performance. ${ }^{11}$ Then, the turbulence phase $\varphi^{\text {tur }}$ can be modeled as

$$
\varphi_{k+1}^{t u r}=a_{1}^{t u r} \varphi_{k}^{t u r}+a_{2}^{t u r} \varphi_{k-1}^{t u r}+v_{k}^{t u r}
$$

where $v^{t u r}$ is gaussean white noise, with variance $\sigma_{v, t u r}^{2}$.

Following the same approach, vibration phases $\varphi^{v i b, i}$, for each one of the $n$ vibrations to be modeled, can be described by a second order autoregresive process, subject to gaussean white noise $v^{v i b, i}$.

$$
\varphi_{k+1}^{v i b, i}=a_{1}^{v i b, i} \varphi_{k}^{v i b, i}+a_{2}^{v i b, i} \varphi_{k-1}^{v i b, i}+v_{k}^{v i b, i}
$$


Values for $a_{1}$ and $a_{2}$ for each AR2 model are obtained by defining a second order continuous-time transfer function with natural frequency $\omega_{o}$ and damping ratio $\zeta$ for each vibration phase. ${ }^{12}$ By matching pole location, $a_{1}$ and $a_{2}$ are given as functions of $\omega_{o}, \zeta$ and the time step for the control loop $T$.

$$
\begin{aligned}
& a_{1}=2 e^{-\zeta \omega_{o} T} \cos \left(\omega_{o} T \sqrt{1-\zeta^{2}}\right) \\
& a_{2}=-e^{-2 \zeta \omega_{o} T}
\end{aligned}
$$

Turbulence and vibration phases, therefore, are determined by their natural frequency $\omega_{o}$, damping ratio $\zeta$ (resonant for vibration peaks) and noise variance $\sigma_{v}^{2}$.

It is possible to identify vibration peaks on the total disturbance phase PSD obtained from the output PSD. Each one of these can be associated to an AR2 resonant process. These will be the vibration phases $\varphi^{v i b, i}$.

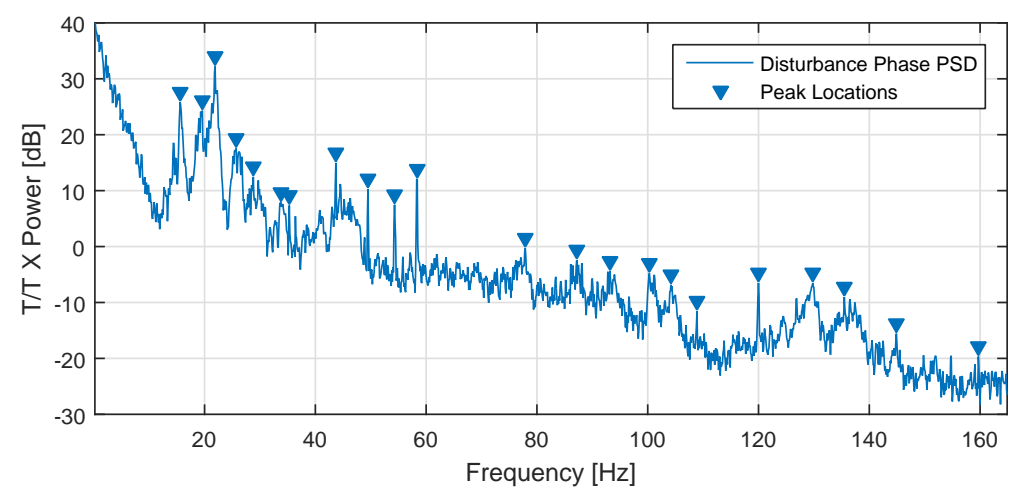

Figure 14: Peak identification in total disturbance phase PSD estimate.

Through an iterative method, second order autorregresive models are found for each of the detected vibration peaks, defining values for $\omega_{o}, \zeta$ and $\sigma_{v}^{2}$ for each peak. Figure 15 presents two of the AR2 models describing vibration phases.
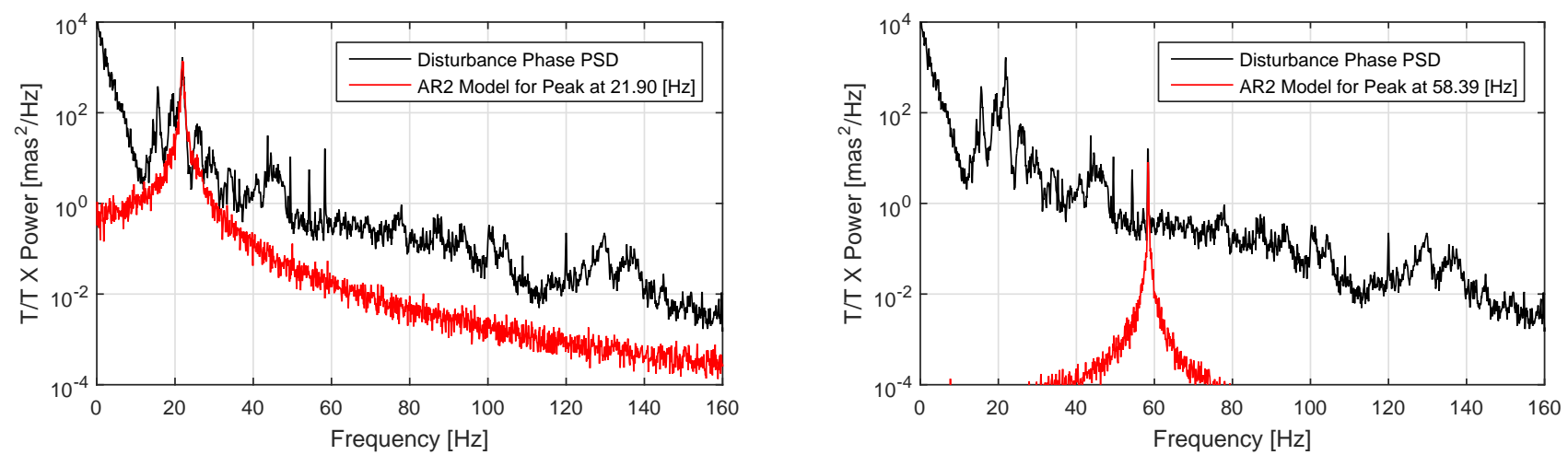

Figure 15: Resonant AR2 models for vibration peaks. Left: AR2 model for peak at $21.90[\mathrm{~Hz}]$. Right: AR2 model for peak at $58.39[\mathrm{~Hz}]$.

The disturbance phase $(\varphi)$ component due to vibrations $\left(\varphi^{v i b, T}=\varphi^{v i b, 1}+\ldots+\varphi^{v i b, n}\right)$ is modeled by the sum of several AR2 models. On the other hand, the turbulence phase $\varphi^{t u r}$ is defined as nonresonant and with a low natural frequency, in order to fit the lower frequencies on the total disturbance phase PSD. 

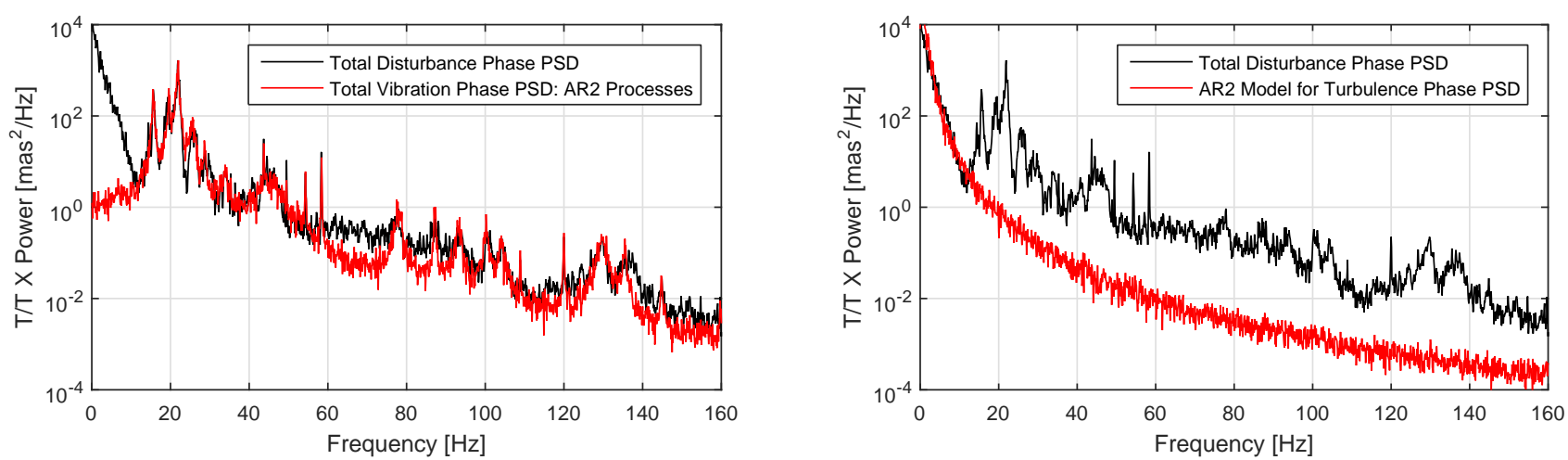

Figure 16: Left: Total vibration phase PSD modeled after the sum of resonant AR2 models. Right: Turbulence phase PSD modeled after the low frequency components on the total disturbance phase PSD.

The complete model for the total disturbance phase PSD is presented in Figure 17.

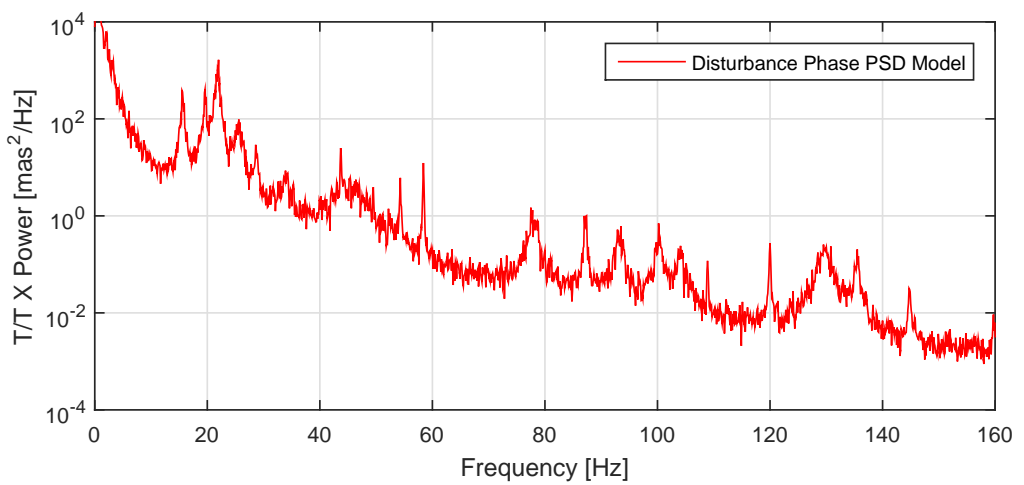

Figure 17: Total disturbance phase PSD estimate, modeled after the sum of nonresonant (for turbulence) and resonant (for vibrations) AR2 models. 


\section{CONTROLLER FOR MAGAO}

A Kalman approach (a special case of the LQG approach ${ }^{6}$ ) is proposed in order to minimize the variance of the slope residuals, including estimates for the disturbance phase temporal evolution.

\subsection{LQG Control Approach}

The model proposed for the MagAO system in Section 3, considering vibration effects, is described by

$$
\begin{aligned}
y_{k} & =w_{k}+D \varphi_{k-1}-D N u_{k-2} \\
\varphi_{k} & =\varphi_{k}^{t u r}+\varphi_{k}^{v i b, 1}+\cdots+\varphi_{k}^{v i b, n} \\
\varphi_{k+1}^{t u r} & =a_{1}^{t u r} \varphi_{k}^{t u r}+a_{2}^{t u r} \varphi_{k-1}^{t u r}+v_{k}^{t u r} \\
\varphi_{k+1}^{v i b, i} & =a_{1}^{v i b, i} \varphi_{k}^{v i b, i}+a_{2}^{v i b, i} \varphi_{k-1}^{v i b, i}+v_{k}^{v i b, i}
\end{aligned}
$$

In order to use an LQG approach for controller design, a state-space representation is required. The state vector can be defined as the phase signals, following their AR2 process model.

$$
\begin{aligned}
\varphi_{k+1} & =a_{1} \varphi_{k}+a_{2} \varphi_{k-1}+v_{k} \\
\Rightarrow\left[\begin{array}{c}
\varphi_{k+1} \\
\varphi_{k}
\end{array}\right] & =\left[\begin{array}{cc}
a_{1} & a_{2} \\
1 & 0
\end{array}\right]\left[\begin{array}{c}
\varphi_{k} \\
\varphi_{k-1}
\end{array}\right]+\left[\begin{array}{c}
v_{k} \\
0
\end{array}\right]
\end{aligned}
$$

Then, the recursive process defining the state vector is:

$$
\begin{aligned}
x_{k+1}= & A x_{k}+v_{k} \\
\Rightarrow x_{k}= & {\left[\begin{array}{lllllll}
\varphi_{k}^{\text {tur }} & \varphi_{k-1}^{\text {tur }} & \varphi_{k}^{v i b, 1} & \varphi_{k-1}^{v i b, 1} & \ldots & \varphi_{k}^{v i b, n} \varphi_{k-1}^{v i b, n}
\end{array}\right]^{T} } \\
A & {\left[\begin{array}{cccccccc}
a_{1}^{\text {tur }} & a_{2}^{\text {tur }} & 0 & 0 & \ldots & 0 & 0 \\
1 & 0 & 0 & 0 & \cdots & 0 & 0 \\
0 & 0 & a_{1}^{v i b, 1} & a_{2}^{v i b, 2} & \ldots & 0 & 0 \\
0 & 0 & 1 & 0 & \ldots & 0 & 0 \\
\vdots & \vdots & \vdots & \vdots & \ddots & \vdots & \vdots \\
0 & 0 & 0 & 0 & \cdots & a_{1}^{v i b, n} & a_{2}^{v i b, n} \\
0 & 0 & 0 & 0 & \cdots & 1 & 0
\end{array}\right] } \\
v_{k}= & {\left[\begin{array}{llllllll}
v_{k}^{\text {tur }} & 0 & v_{k}^{v i b, 1} & 0 & \cdots & v_{k}^{v i b, n} & 0
\end{array}\right]^{T} }
\end{aligned}
$$

The output equation is defined.

$$
\begin{aligned}
y_{k} & =C x_{k}-D N u_{k-2}+w_{k} \\
\Rightarrow C & =\left[\begin{array}{lllllll}
0 & D & 0 & D & \cdots & 0 & D
\end{array}\right]
\end{aligned}
$$

Then, equations (22) and (26) describe the state-space representation for the MagAO system subject to vibration.

It has been proved ${ }^{10}$ than the optimal controller for this system, in terms of the minimization of error variance (considering $\varphi^{r e s}$ as the error signal), is a LQG controller, based on an optimal state estimation through a Kalman filter. ${ }^{6}$

The state prediction equation is defined. 


$$
\begin{aligned}
\hat{x}_{k+1 \mid k} & =A \hat{x}_{k \mid k-1}+L\left(y_{k}-\hat{y}_{k \mid k-1}\right) \\
& =A \hat{x}_{k \mid k-1}+L\left(y_{k}-C \hat{x}_{k \mid k-1}+D N u_{k-2}\right)
\end{aligned}
$$

where $L$ is the stationary Kalman gain. Defining measuremente noise variance $\sigma_{w}^{2}, L$ is computed by

$$
L=A P C^{T}\left(C P C^{T}+\sigma_{w}^{2}\right)^{-1}
$$

$P$ corresponds to the solution for the following discrete-time algebraic Ricatti equation

$$
P=A P A^{T}+A P C^{T}\left(C P C^{T}+\sigma_{w}^{2}\right)^{-1} C P A^{T}+R_{v}
$$

$R_{v}$ is the phase noise covariance matrix, defined as

$$
R_{v}=\left[\begin{array}{ccccccc}
\sigma_{v, \text { tur }}^{2} & 0 & 0 & 0 & \cdots & 0 & 0 \\
0 & 0 & 0 & 0 & \cdots & 0 & 0 \\
0 & 0 & \sigma_{v, v i b, 1}^{2} & 0 & \cdots & 0 & 0 \\
0 & 0 & 0 & 0 & \cdots & 0 & 0 \\
\vdots & \vdots & \vdots & \vdots & \ddots & \vdots & \vdots \\
0 & 0 & 0 & 0 & \cdots & \sigma_{v, v i b, n}^{2} & 0 \\
0 & 0 & 0 & 0 & \cdots & 0 & 0
\end{array}\right]
$$

The control design objective is to minimize error variance, being $\varphi^{\text {res }}$ the error signal. Then, the cost function $J_{L Q R}$ is defined by

$$
\begin{aligned}
J_{L Q R} & =\sigma_{\text {res }}^{2}=E\left\{\sum_{k=0}^{N}\left(\left(\varphi_{k}^{\text {res }}\right)^{T}\left(\varphi_{k}^{\text {res }}\right)\right)\right\} \\
& =E\left\{\sum_{k=0}^{N}\left(\left(\varphi_{k}-\varphi_{k}^{\text {cor }}\right)^{T}\left(\varphi_{k}-\varphi_{k}^{\text {cor }}\right)\right)\right\} \\
& =E\left\{\sum_{k=0}^{N}\left(\left(\left[\begin{array}{lllll}
1 & 0 & 1 & 0 & \cdots
\end{array}\right] x_{k}-N u_{k-1}\right)^{T}\left(\left[\begin{array}{lllll}
1 & 0 & 1 & 0 & \ldots
\end{array}\right] x_{k}-N u_{k-1}\right)\right)\right\}
\end{aligned}
$$

For each step $k$, an estimation for the following state vector $\hat{x}_{k+1 \mid k}$ is available. Therefore, $u_{k}$ is defined in order to minimize the cost function in (32).

$$
\hat{u}_{k}=N^{-1}\left[\begin{array}{lllll}
1 & 0 & 1 & 0 & \cdots
\end{array}\right] \hat{x}_{k+1 \mid k}
$$

\subsection{Simulation Results}

In order to observe and compare integrator-based controller performance and LQG control performance, an estimate for the total disturbance phase is desirable. By interpolating the time-domain output data measured on-sky and inverting the closed-loop transfer function, an estimate for the temporal evolution of the phase modal amplitude $\varphi$ is obtained.

The phase PSD obtained from the time-domain phase estimate and the previously obtained phase PSD estimate presented in Figure 13b differ slightly due to the interpolation process, specially on higher frequencies.

The AO loop is simulated, using both an integrator-based controller and the LQG control method presented in section 4.1. Graphical results are presented in Figure 18. 


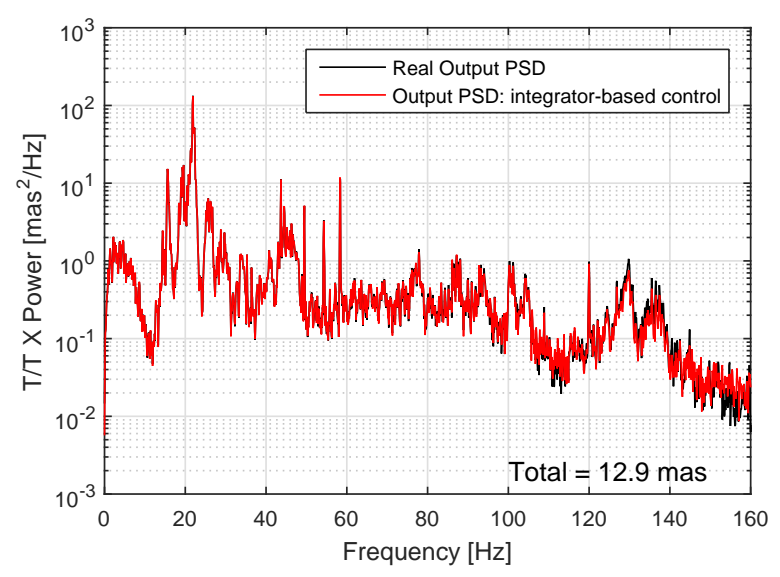

(a) Using an integrator-based controller.

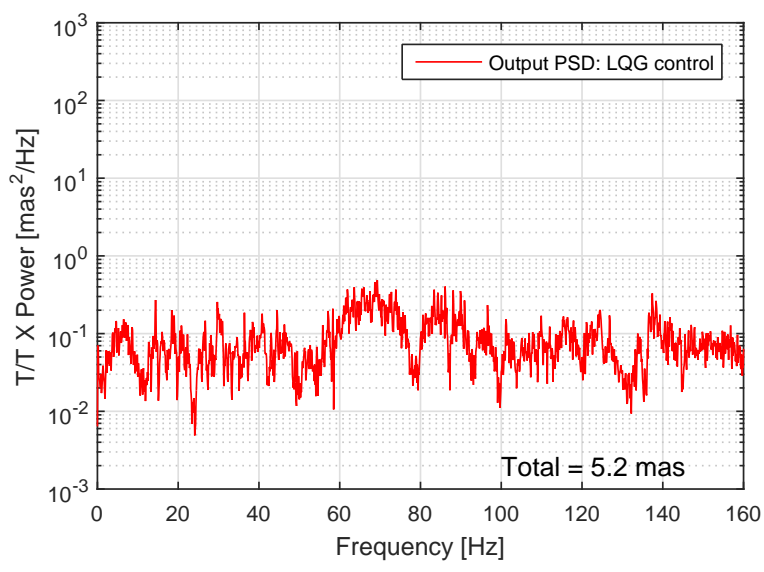

(b) Using an LQG control approach.

Figure 18: Output PSDs obtained from AO loop simulation. RMS values are presented for each output signal.

Figure 18a presents both the real output PSD and the simulation result for the output PSD using an integrator-based controller. These signals should be equal, attributing the difference at higher frequencies to the interpolation process to obtain the time-domain phase estimate used during simulation.

Incorporation of AR2 models for turbulence and vibration phases in the LQG control design allows to counter these effects on the output's frequencial components, as shown on Figure 18b. Thus, the output's RMS value for this control alternative is significantly lower than the integrator approach.

The LQG control is designed from a disturbance phase PSD estimate using Test A output data (see Table 1). Therefore, it takes into account only vibration components detected in Test A.

Test D data (with primary fans on, primary pump on and louvers open) is used to test the LQG control alternative. A new time-domain phase estimate is obtained for Test D, and both an integrator-based controller and the LQG control method are simulated for the AO loop. Graphical results are presented in Figure 19.

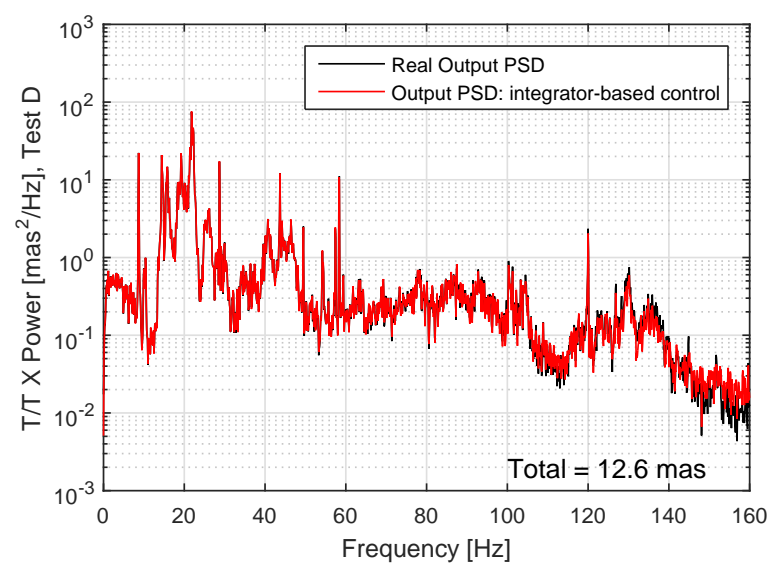

(a) Using an integrator-based controller.

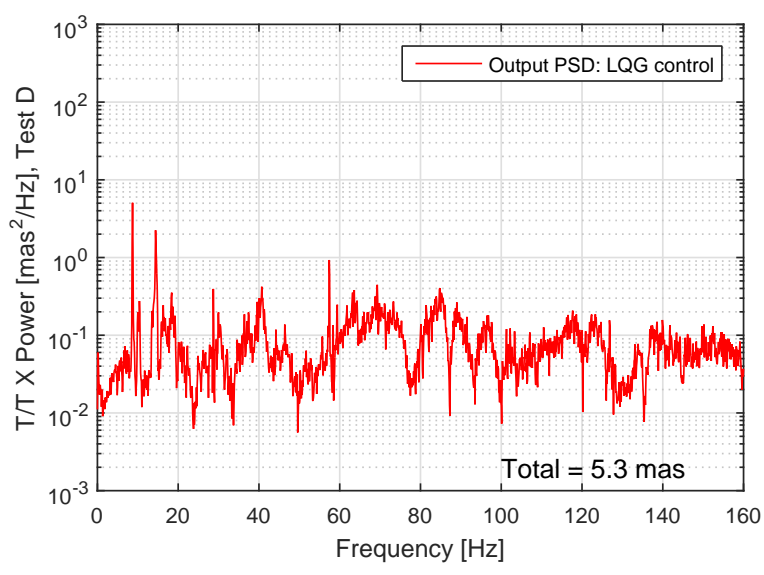

(b) Using an LQG control approach.

Figure 19: Output PSDs obtained from AO loop simulation, for Test D. RMS values are presented for each output signal.

Since the LQG control approach designed for Test A is used in Test D data, it does not take into account the new vibration effects caused by the instrumentation, as observed in Figure 19b. Therefore, during on-sky 
measurements, the disturbance phase's models should be constantly updated, in order to include new possible frequency components, as the ones induced by the system's instrumentation.

\subsection{Predictive Control Approach}

LQG control previously presented for the AO loop is the optimal control scheme in terms of the minimization of residual phase variance. However, this is guaranteed only within the linearity of the system, exempt from constraints.

The model predictive control (MPC) approach is useful when imposing constraints to the system's signals.

As an example, Figure 20 presents the grafical results for output PSDs when the LQG controller is applied and the controller output $u$ is constrained.

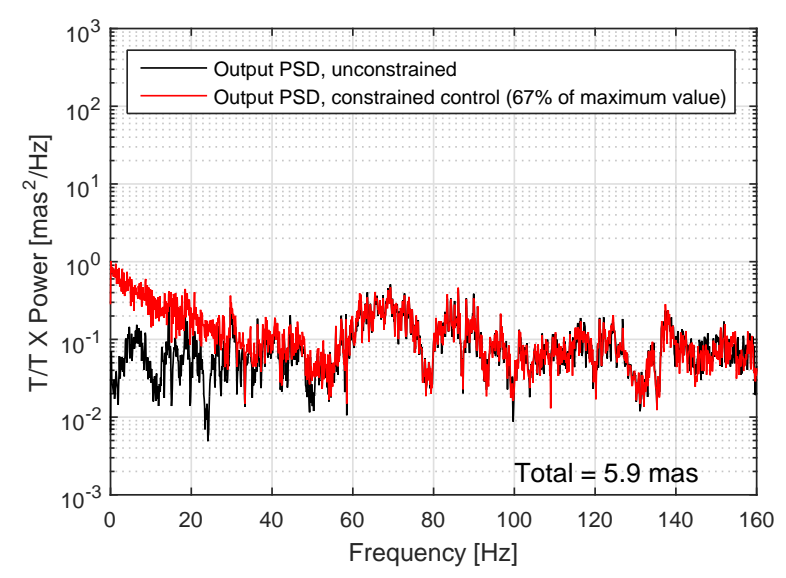

(a) Controller output limited by $67 \%$ of its unconstrained maximum value.

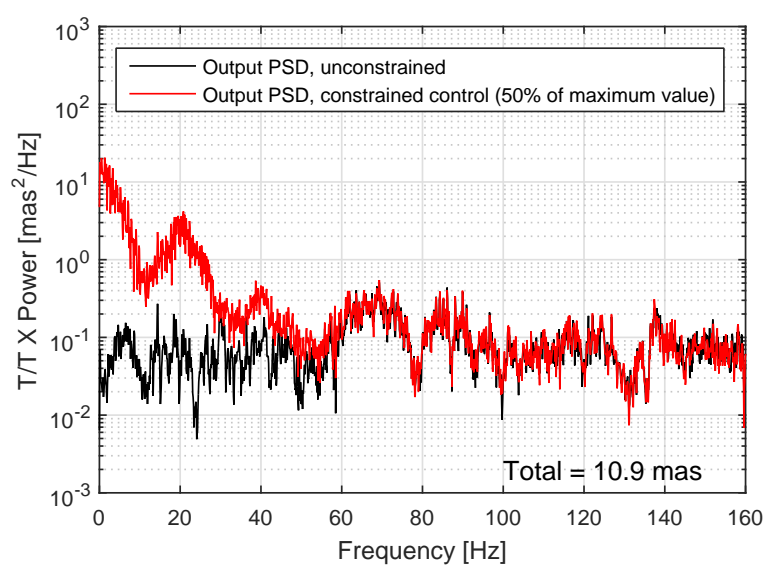

(b) Controller output limited by $50 \%$ of its unconstrained maximum value.

Figure 20: Effect of constrained controller output on LQG control performance. RMS values are presented for each case.

Model predictive control is often used for control systems subject to saturation. For this method, the control objetive is not only to minimize the residual slope variance at the present step, but also to use an estimator for the residual slope and minimize its variance in the future.

The MPC cost function is presented.

$$
\begin{aligned}
J_{M P C} & =E\left\{\sum_{k=\ell}^{\ell+N}\left(\left(\varphi_{k}^{r e s}\right)^{T}\left(\varphi_{k}^{r e s}\right)\right)\right\}=E\left\{\sum_{k=\ell}^{\ell+N} \hat{J}_{M P C, k}\right\} \\
\hat{J}_{M P C, k} & =\left(\left[\begin{array}{lllll}
1 & 0 & 1 & 0 & \cdots
\end{array}\right] x_{k}-N u_{k-1}\right)^{T}\left(\left[\begin{array}{lllll}
1 & 0 & 1 & 0 & \cdots
\end{array}\right] x_{k}-N u_{k-1}\right)
\end{aligned}
$$

The control objective for MPC is to find a set of actuation signals $u_{\ell}, \ldots, u_{\ell+N}$ which minimizes the cost function $J_{M P C}$, given an initial state vector $x_{\ell}$.

The proposed model defines the disturbance phase modal amplitudes as state vectors, through autorregresive models. Then, $\hat{x}_{k}$ (for $k \geq \ell$ ) only depends on previous values for the state vector, and specifically, on $x_{\ell}$. Therefore, $\hat{J}_{M P C, k}$ depends only on the controller output at the previous step $\left(u_{k-1}\right)$ and the initial state vector value $x_{\ell}$.

In conclusion, the $J_{M P C}$ minimization problem is reduced into minimizing each $J_{M P C, k}$ separately, thus giving the same result as the LQG approach. 
In order to benefit from a model predictive control approach, the disturbance phases must be defined not only by the previous disturbance phases (regressive model), but also by an external input signal $u$.

It is known that certain instruments of the MagAO system (primary fans, primary pump, louvers) are responsable for the presence of vibration peaks on the captured data PSD. A nonlinear model which includes the status of these instruments and their influence on the disturbance phase could benefit from a predictive control approach.

\section{CONCLUSIONS}

We have presented an optimal closed-loop LQG control law for the MagAO system, based on a state-space model obtained from the spectral analysis of sensor data. The control approach here suggested has been previously tested and implemented on different $\mathrm{AO}$ systems. The main improvement of this technique over integrator-based control (even with an optimized controller gain) is integration of temporal evolution of turbulence and vibrations, through second order autorregresive models.

Due to the limited data available from the MagAO loop, the model here obtained can be improved with different captured data or knowledge of the dynamics of instruments involved. With a precise model available, and the design of an optimal controller (the LQG controller analysed in this document or other alternatives), the next step would be to put the new controller designs into practice and, thus, implementing vibration mitigation techniques on the system.

\section{ACKNOWLEDGMENTS}

The research for this paper couldn't have been possible without the support and generosity of the MagAO Team: Dr. Laird Close, Dr. Jared Males and Dr. Katie Morzinski, who have allow us to work and investigate the MagAO system alongside them during the Winter Research Program 2015 at the University of Arizona.

I gratefully acknowledge Universidad Técnica Federico Santa María, as well as the financial support by CONICYT, Chile. This work was supported by the Advanced Center for Electrical and Electronic Engineering, AC3E, Basal Project FB0008, CONICYT, with partial support by the Fondef IDeA IT15I10041 grant.

\section{REFERENCES}

[1] K. Morzinski, L. Close, J. Males, D. Kopon, P. Hinz, S. Esposito, A. Riccardi, A. Puglisi, E. Pinna, R. Briguglio, M. Xompero, F. Quirós-Pacheco, V. Bailey, K. Follette, T. Rodigas, Y. Wu, C. Arcidiacono, J. Argomedo, L. Busoni, T. Hare, A. Uomoto, and A. Weinberger, MagAO: Status and on-sky performance of the Magellan adaptive optics system, vol. 9148. SPIE, 2014.

[2] L. Close, J. Males, D. Kopon, V. Gasho, K. Follette, P. Hinz, K. Morzinski, A. Uomoto, T. Hare, A. Riccardi, S. Esposito, A. Puglisi, E. Pinna, L. Busoni, C. Arcidiacono, M. Xompero, R. Briguglio, F. Quiros-Pacheco, and J. Argomedo, First closed-loop visible AO test results for the advanced adaptive secondary AO system for the Magellan Telescope: MagAO's performance and status, vol. 8447. 2012.

[3] S. Zuniga, J. Garces, L. M. Close, J. R. Males, K. M. Morzinski, P. Escarate, and M. Castro, "Vibrations in magao: analysis of on-sky data and future challenges in vibrations mitigation," in Imaging and Applied Optics 2015, Imaging and Applied Optics 2015, p. AOTh1D.4, Optical Society of America, 2015.

[4] J. Garces, S. Zuniga, L. M. Close, J. R. Males, K. M. Morzinski, P. Escarate, and M. Castro, "Vibrations in magao: resonance sources identification through frequency-based analysis," in Imaging and Applied Optics 2015, Imaging and Applied Optics 2015, p. AOTh1D.3, Optical Society of America, 2015.

[5] J. Males, L. Close, O. Guyon, K. Morzinski, A. Puglisi, P. Hinz, K. Follette, J. Monnier, V. Tolls, T. Rodigas, A. Weinberger, A. Boss, D. Kopon, Y. Wu, S. Esposito, A. Riccardi, M. Xompero, R. Briguglio, and E. Pinna, Direct imaging of exoplanets in the habitable zone with adaptive optics, vol. 9148. SPIE, 2014.

[6] A. Guesalaga, B. Neichel, F. Rigaut, J. Osborn, and D. Guzman, "Comparison of vibration mitigation controllers for adaptive optics systems," Appl. Opt. 51, pp. 4520-4535, Jul 2012. 
[7] G. Sivo, C. Kulcsár, J.-M. Conan, H.-F. Raynaud, Éric Gendron, A. Basden, F. Vidal, T. Morris, S. Meimon, C. Petit, D. Gratadour, O. Martin, Z. Hubert, A. Sevin, D. Perret, F. Chemla, G. Rousset, N. Dipper, G. Talbot, E. Younger, R. Myers, D. Henry, S. Todd, D. Atkinson, C. Dickson, and A. Longmore, "First onsky scao validation of full lqg control with vibration mitigation on the canary pathfinder," Opt. Express 22, pp. 23565-23591, Sep 2014.

[8] P. D. Welch, "The use of fast fourier transform for the estimation of power spectra: A method based on time averaging over short, modified periodograms," IEEE Transactions on audio and electroacoustics $\mathbf{1 5}(2)$, pp. 70-73, 1967.

[9] E. Gendron and P. Lena, "Astronomical adaptive optics. 1: Modal control optimization," aap 291, pp. 337347, Nov. 1994.

[10] B. L. Roux, C. Kulcsár, L. M. Mugnier, T. Fusco, H.-F. Raynaud, and J.-M. Conan, "Optimal control law for classical and multiconjugate adaptive optics," J. Opt. Soc. Am. A 21, pp. 1261-1276, Jul 2004.

[11] C. Kulcsár, H.-F. Raynaud, J.-M. Conan, C. Correia, and C. Petit, "Control design and turbulent phase models in adaptive optics: A state-space interpretation," in Frontiers in Optics 2009/Laser Science XXV/Fall 2009 OSA Optics 83 Photonics Technical Digest, Frontiers in Optics 2009/Laser Science XXV/Fall 2009 OSA Optics \& Photonics Technical Digest, p. AOWB1, Optical Society of America, 2009.

[12] C. Petit, J.-M. Conan, C. Kulcsár, H.-F. Raynaud, and T. Fusco, "First laboratory validation of vibration filtering with lqg control law for adaptive optics," Opt. Express 16, pp. 87-97, Jan 2008. 